Article

\title{
Single-Grain Gate-All-Around Si Nanowire FET Using Low-Thermal-Budget Processes for Monolithic Three-Dimensional Integrated Circuits
}

\author{
Tung-Ying Hsieh ${ }^{1,2}$, Ping-Yi Hsieh ${ }^{3}{ }^{(0)}$, Chih-Chao Yang ${ }^{3, *}$, Chang-Hong Shen ${ }^{3}$, Jia-Min Shieh ${ }^{3}$, \\ Wen-Kuan $\mathrm{Yeh}^{3}$ and Meng-Chyi $\mathrm{Wu}^{1}$ \\ 1 Institute of Electronics Engineering, National Tsing Hua University, Hsinchu 30013, Taiwan; \\ tyhsieh@narlabs.org.tw (T.-Y.H.); mcwu@ee.nthu.edu.tw (M.-C.W.) \\ 2 National Applied Research Laboratories, 3F, No. 106, Ho Ping E. Rd., Sec. 2, Taipei City 10622, Taiwan \\ 3 Taiwan Semiconductor Research Institute, No.26, Prosperity Road 1, Hsinchu 30013, Taiwan; \\ 1805047@narlabs.org.tw (P.-Y.H.); chshen@narlabs.org.tw (C.-H.S.); jmshieh@narlabs.org.tw (J.-M.S.); \\ 1305023@narlabs.org.tw (W.-K.Y.) \\ * Correspondence: samyang@narlabs.org.tw; Tel.: +886-3-5276110 (ext. 7565)
}

Received: 4 July 2020; Accepted: 30 July 2020; Published: 30 July 2020

\begin{abstract}
We introduce a single-grain gate-all-around (GAA) Si nanowire (NW) FET using the locationcontrolled-grain technique and several innovative low-thermal budget processes, including green nanosecond laser crystallization, far-infrared laser annealing, and hybrid laser-assisted salicidation, that keep the substrate temperature $\left(\mathrm{T}_{\text {sub }}\right.$ ) lower than $400{ }^{\circ} \mathrm{C}$ for monolithic three-dimensional integrated circuits (3D-ICs). The detailed process verification of a low-defect GAA nanowire and electrical characteristics were investigated in this article. The GAA Si NW FETs, which were intentionally fabricated within the controlled Si grain, exhibit a steeper subthreshold swing (S.S.) of about $65 \mathrm{mV} / \mathrm{dec}$. , higher driving currents of $327 \mu \mathrm{A} / \mu \mathrm{m}$ (n-type) and $297 \mu \mathrm{A} / \mu \mathrm{m}$ (p-type) @ $\mathrm{V}_{\text {th }} \pm 0.8 \mathrm{~V}$, and higher $\mathrm{I}_{\mathrm{on}} / \mathrm{I}_{\text {off }}\left(>10^{5} @\left|\mathrm{~V}_{\mathrm{d}}\right|=1 \mathrm{~V}\right)$ and have a narrower electrical property distribution. In addition, the proposed Si NW FETs with a GAA structure were found to be less sensitive to $V_{\text {th }}$ roll-off and S.S. degradation compared to the omega $(\Omega)$-gate Si FETs. It enables ultrahigh-density sequentially stackable integrated circuits with superior performance and low power consumption for future mobile and neuromorphic applications.
\end{abstract}

Keywords: monolithic 3D; gate-all-around; nanowire FET; low-thermal budget; location-controlledgrain; laser crystallization; laser activation; laser-assisted salicidation; low power consumption

\section{Introduction}

To catch up to Moore's law, and to benefit from a faster computing speed and economical chip, there are two approaches [1,2] followed and adopted in the semiconductor industry: one is "more Moore" which means keep downsizing the dimension of the transistor using a new device structure or non-silicon materials; the other is "more than Moore" which introduces a heterogeneous integration concept in the out-of-plane direction. The latter is also known as three-dimensional integrated circuits (3D-ICs). Although through silicon via (TSV) technology [3] is the dominating process in modern 3D-ICs, monolithic 3D-ICs $[4,5]$ have drawn increasing attention in recent years which can offer additional transistor layers using either front end of line (FEOL) or back end of line (BEOL) processes for integrated circuits, and thereby provide several advantages, such as the reduction of latency, flexibility, high connectivity, and low power consumption [6].

Low-temperature polycrystalline-silicon (LTPS) thin-film transistors (TFTs) fabricated by excimer laser crystallization (ELC) drew tremendous attention in the 1980s $[7,8]$ for their great potential in realizing three-dimensional integrated circuits (3D-ICs). However, the random grain size distribution, 
the unpredictable grain boundary, and the narrow process window to prevent damages on the bottom tier become ineluctable issues [9,10]. To date, there has been several researches aiming to develop sequentially 3D stackable techniques using a layer transfer method, namely SmartCut ${ }^{\mathrm{TM}}$ with semiconductoron-insulator wafers, e.g., SOI, GOI, or III-V-OI [11,12]. Although this technique could provide better channel quality, it still needs to face the potential high-temperature annealing process of 3D stackable transistor manufacturing. In addition, the process complexity and costly investment may hinder its widespread development. In contrast, there are several groups trying to modify the recrystallization process to prevent the grain boundary effect and to minimize the number of grain boundaries within the active region of a device. One of them is the $\mu$-Czochralski process that tries to control the grain location so then devices can be manufactured within the grain [13-15]. However, the thick and rough channel is not appropriate for modern nano-electronics. The main task is to develop a thin and high-crystallinity channel and further low-thermal budget processes for sequentially 3D stackable devices with high performance and low variability.

In this article, we adopted a $\mu$-Czochralski process using pulse laser crystallization followed by a chemical mechanical polish (CMP) and surface modification steps to fabricate a thin and high-crystallinity location-controlled grain (LCG) Si nanowire channel for its high positioning accuracy, predictability, and compatibility with our present process. A gate-all-around configuration to detach the Si and buried $\mathrm{SiO}_{2}$ for a nano-scale transistor, which is much smaller than the grain size of the Si channel, integrated with several innovative low-thermal budget processes was demonstrated. The single-grain GAA Si NW FETs and processes earn not only high performance and low variability but the extremely scaled NW dimension, which, in turn, enables the application for advanced monolithic 3D-ICs.

\section{Materials and Methods}

The process flow of the monolithic 3D single-grain gate-all-around Si nanowire FET is depicted in Figure 1. The key of fabricating such a sequentially 3D stackable transistor or further integrated circuit lies in developing low-thermal budget processes, which means the substrate temperature should not be higher than $400{ }^{\circ} \mathrm{C}$ to keep the metal interconnect reliable and to be compatible with BEOL processes $[16,17]$. To prepare a high-crystallinity and LCG Si channel, we use a shaped e-beam direct write lithography system and inductively coupled plasma (ICP) etching to create periodic cooling holes on buried oxide as a grain filter before deposing a conformal and 150-nm-thick amorphous Si (a-Si) film. Afterwards, the a-Si film was recrystallized and phase-transformed to a location-controlled and large-grain polycrystalline Si film by Nd:YAG diode-pumped solid-state (DPSS) continuous wave green nanosecond laser annealing (GNS-LA) (Figure 1a). The wavelength, pulse width, scanning speed, beam size, and power of the laser are $\lambda=532 \mathrm{~nm}, 13 \mathrm{~ns}, 25 \mathrm{~cm} / \mathrm{s}, 2 \mathrm{~mm} \times 40 \mu \mathrm{m}$, and $5.5 \mathrm{~W}$, respectively. To remove the surface defects caused by the laser process and to improve the overall channel uniformity, the CMP was introduced to thin the thick and large-grain polycrystalline Si film down to below $50 \mathrm{~nm}$ (Figure 1b). Afterward, an NW was preliminary defined by a shaped e-beam direct write lithography system and the ICP etching process.

A whole chemical process was then proceeded to suspend $\mathrm{NW}$ in this article as follows: a. soak into two mixture solutions $\left(\mathrm{NH}_{4} \mathrm{OH}: \mathrm{H}_{2} \mathrm{O}_{2}: \mathrm{H}_{2} \mathrm{O}=1: 4: 20\right.$ and $\left.\mathrm{HCl}: \mathrm{H}_{2} \mathrm{O}_{2}: \mathrm{H}_{2} \mathrm{O}=1: 1: 6\right)$ at $75^{\circ} \mathrm{C}$ for $10 \mathrm{~min}$ to remove polymer residues and particles caused by the photoresist stripping process (Figure 1c); b. immerse into pure $\mathrm{H}_{2} \mathrm{O}_{2}$ as a strong oxidizing agent for 3 minutes at $100^{\circ} \mathrm{C}$ to grow sacrificial oxide on the Si surface (Figure 1d); c. suspend the Si NW by removing the sacrificial oxide using dilute HF solution ( $\left.\mathrm{HF}: \mathrm{H}_{2} \mathrm{O}=1: 10\right)$ for 5 min (Figure 1e).

The gate stack was fabricated by 3nm-thick $\mathrm{HfO}_{2}$ and 50 -nm-thick TaN metal layers using atomic layer deposition (ALD) for its excellent step coverage and self-limiting nature around the NW as high-K gate dielectric and metal electrode (HK/MG), respectively (Figure 1f). The gate length of the Si NW FET was defined by shaped e-beam direct write lithography ICP etching process. The exposed channel region was then doped with $\mathrm{P}^{31+}$ or $\mathrm{BF}_{2}{ }^{49+}$ at a dosage of $5 \times 10^{15} \mathrm{~cm}^{-2}(10 \mathrm{KeV})$ for N-type or P-type MOSFETs through self-aligned ion implantation and was activated by a $\mathrm{CO}_{2}$ FIR-LA to form a highly-activated, 
abrupt, and ultra-shallow junction source and drain regions (Figure $1 \mathrm{~g}$ ). The wavelength, power, and substrate temperature of FIR-LA were $10.6 \mu \mathrm{m}, 125 \mathrm{~W}$, and $350^{\circ} \mathrm{C}$, respectively.

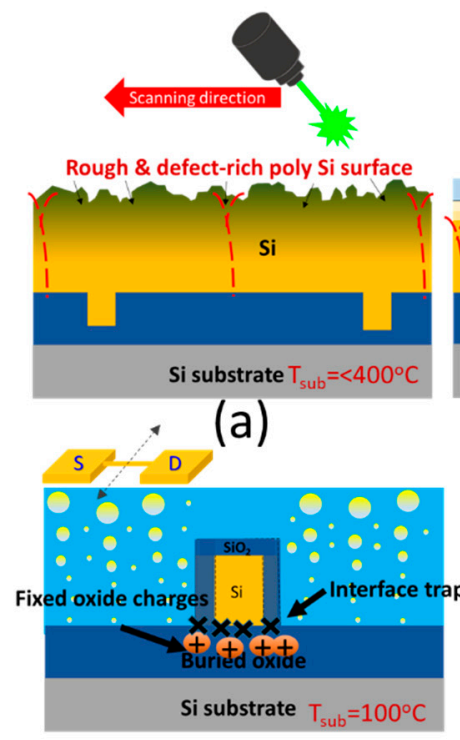

(d)

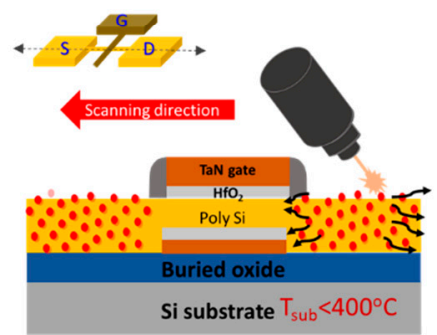

(g)

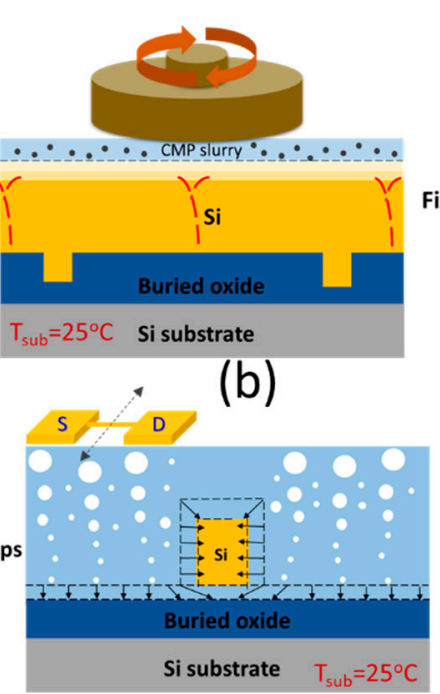

(e)

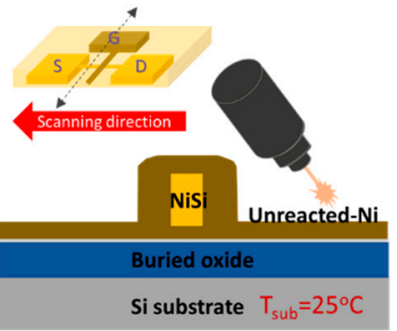

(h)

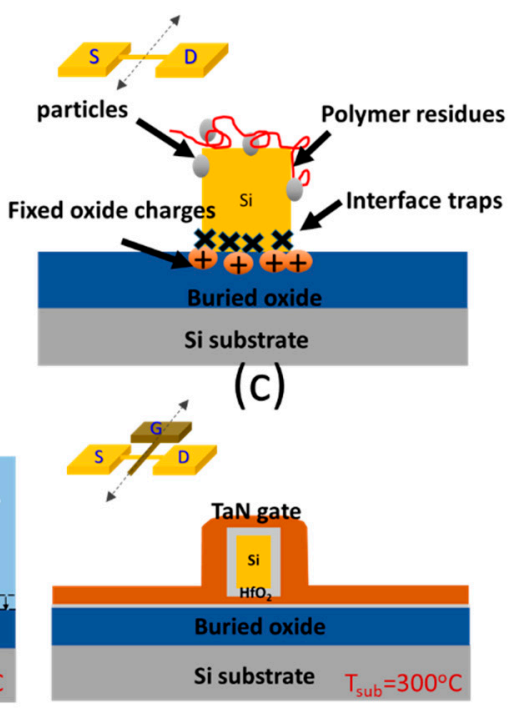

(f)

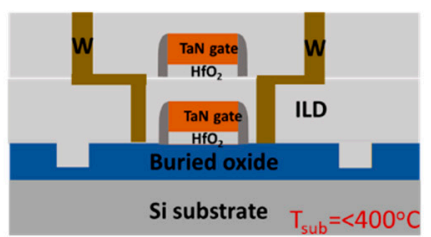

(i)

Figure 1. Schematics of process steps for 3D stackable single-grain gate-all-around (GAA) Si nanowire (NW) FET: (a) a-Si film was crystallized by GNS-LA; (b) the chemical mechanical polishing (CMP); (c) NW with defects was preliminary defined by lithography and ICP etching process in a Si gain; (d) soak into two mixture solutions to remove polymer residues and particles and immerse into pure $\mathrm{H}_{2} \mathrm{O}_{2}$ to form sacrificial oxide on the Si NW surface; (e) suspend the Si NW by removing the sacrificial oxide using dilute HF solution; (f) high-K/metal gate (HK/MG) stack fabricated by ALD; (g) implantation and far-infrared laser annealing (FIR-LA) for dopant activation, (h) hybrid laser-assisted self-aligned silicide and (i) interlayer dielectric layer (ILD) deposition and following standard metallization using tungsten (W) interconnect.

Moreover, a two-step hybrid laser-assisted self-aligned silicide (Salicide) was also adopted to replace the conventional high-temperature Salicide process [18,19]. In the process, a 10-nm-thin Ni film was sputtered on the NW surface after $\mathrm{SiN}_{\mathrm{x}}$ spacer formation. The first-step annealing was carried out at $250{ }^{\circ} \mathrm{C}$ for $30 \mathrm{~s}$ in a rapid thermal annealing (RTA) system to form a high-resistivity $\mathrm{Ni}_{2} \mathrm{Si}$ phase. After removing the unreacted $\mathrm{Ni}$ by soaking into a $1: 10 \mathrm{HNO}_{3}$ solution at $50{ }^{\circ} \mathrm{C}$ for $600 \mathrm{~s}$, the second-step annealing was followed by a $\mathrm{CO}_{2}$ FIR-LA with $100 \mathrm{~W}$ laser power at room temperature to transform the $\mathrm{Ni}_{2} \mathrm{Si}$ phase into a low-resistivity NiSi phase (Figure $1 \mathrm{~h}$ ).

After performing all the low-thermal budget processes to fabricate the single-grain GAA Si NW FETs, $\mathrm{SiO}_{2}$ deposition as an interlayer dielectric layer (ILD) followed by a standard metallization process using tungsten $(\mathrm{W})$ metal were then proceeded to accomplish the first stacked device tier (Figure 1i). A two-tier monolithic 3D-IC can be simply realized by repeating the whole low-thermal budget process $\left(\mathrm{T}_{\text {sub }}<400{ }^{\circ} \mathrm{C}\right)$ (Figure $\left.2 \mathrm{a}, \mathrm{b}\right)$. 


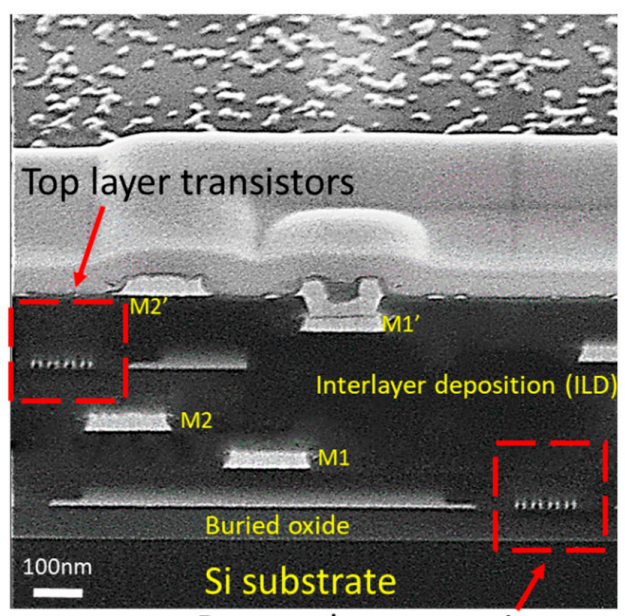

Bottom layer transistors

(a)

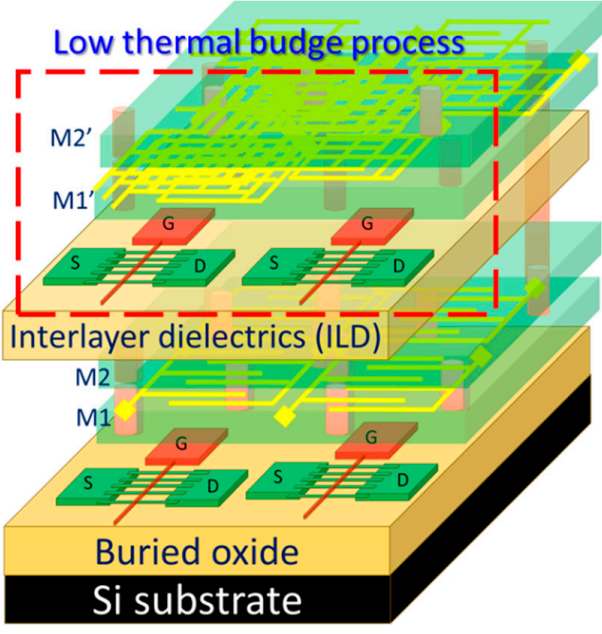

(b)

Figure 2. (a) SEM image of a 3D sequential integration with two stacking tiers and each tier has a two-layer W interconnect (M1-M2). (b) Schematic illusion of a monolith three-dimensional integrated circuit (3DIC) using single-grain GAA Si NW FETs.

\section{Results and Discussion}

\subsection{High-Crystallinity and Controlled-Grain Si Film}

Figure 3a depicts an SEM image of a random grain growth Si channel on the buried oxide after GNS-LA. Apparently, without any specific structure of the surface or crystallization method, the grain size and grain boundary were randomly distributed. The grain boundary in the Si channel may have unreacted dangling bonds or hydrogen-passivated -Si-H bonds that decrease the carrier mobility and degrade the device performance [20-22]. In contrast, Figure 3b shows an SEM image of a Si channel with a regular Si matrix using the location-controlled-grain technique, as illustrated in the inset. The cooling hole acts as a grain filter for lateral grain growth during the melted and quenched laser recrystallization process. The grain boundaries appeared while the neighbor grains were met together. This can be clearly observed after CMP and SEECO etch as shown in Figure 3c. The GAA Si NW FET was designed and intentionally located within a single grain to avoid overlapping the grain boundaries (Figure 3d) which can guarantee better device performance and less output characteristic variation for the monolithic 3DIC design $[23,24]$.

Moreover, a quantitative grain size distribution analysis was conducted by the ImageJ software (Figure 3e). Compared to an approximate grain size around $900 \mathrm{~nm}$ extracted from the location-controlledgrain Si film, the Si channel without periodic cooling holes exhibits a broad grain size distribution with a standard deviation of $228.3 \mathrm{~nm}$ and an average grain size of $631.5 \mathrm{~nm}$ from the statistical data in the Figure 3e. The smallest grain size was only about $100 \mathrm{~nm}$ that was slightly larger than the size of the GAA Si NW FET. This raises the probability of nano-devices located on the grain boundary. This result suggests that the introduction of periodic cooling holes on buried oxide before the laser crystallization process can effectively achieve a location-controlled-grain Si film.

In addition to the $\mu$-Czochralski process, the CMP process was also adopted for fabricating a thin and uniform Si channel after the short-pulse GNS-LA. As a result, the CMP not only eliminates the surface roughness but also removes defect and nanocrystalline Si phase layer on the uneven channel surface. Figure $3 \mathrm{f}$ shows the AFM images and Raman spectroscopy. The surface roughness of the $\mathrm{Si}$ channel dropped from 14.6 to $1.17 \mathrm{~nm}$ and the crystallinity was improved while the channel thickness was decreased from 150 to $20 \mathrm{~nm}$. Evidentially, a Raman peak at $520 \mathrm{~cm}^{-1}$ representing a nanocrystalline Si signal vanished after the CMP process, revealing the poor crystallinity on the surface due to the slower cooling rate in the interfacial region [25]. 


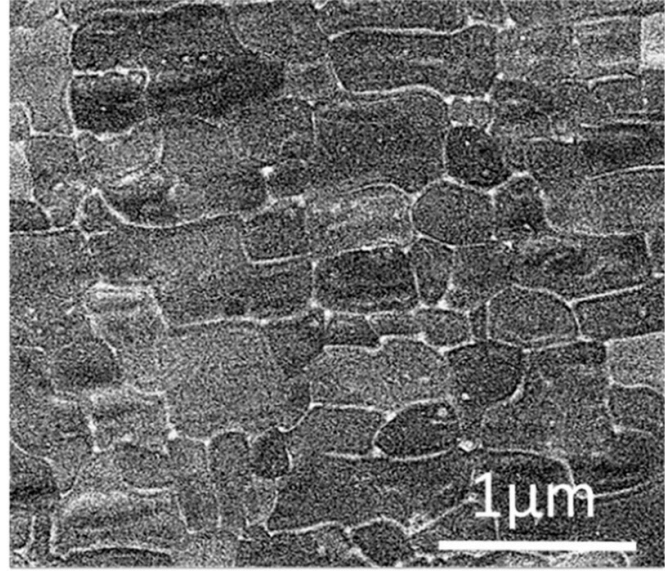

(a)

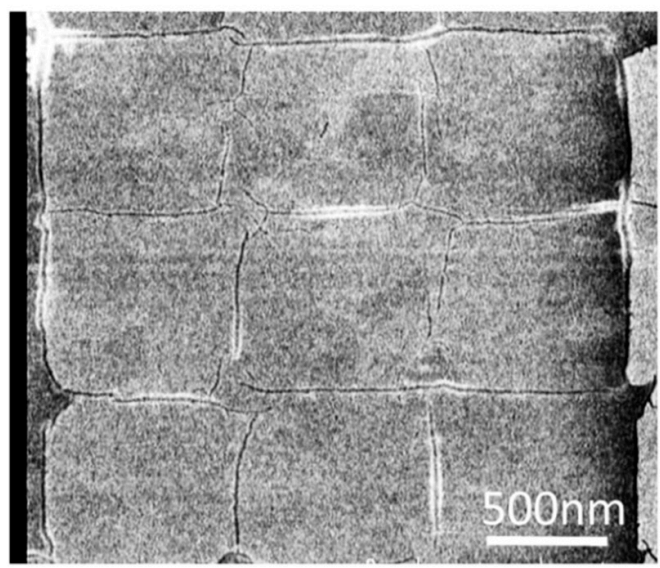

(c)

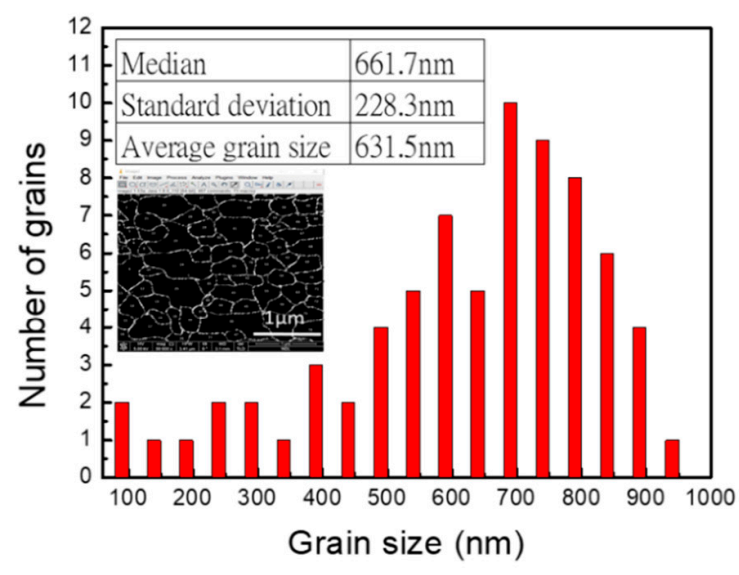

(e)

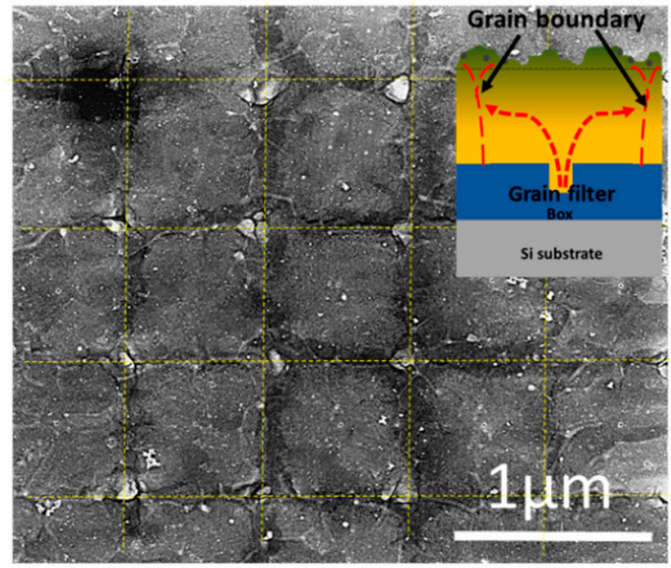

(b)

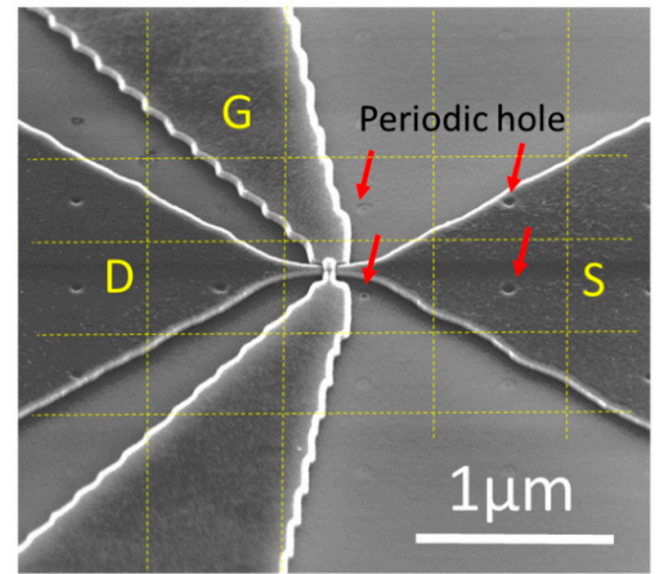

(d)

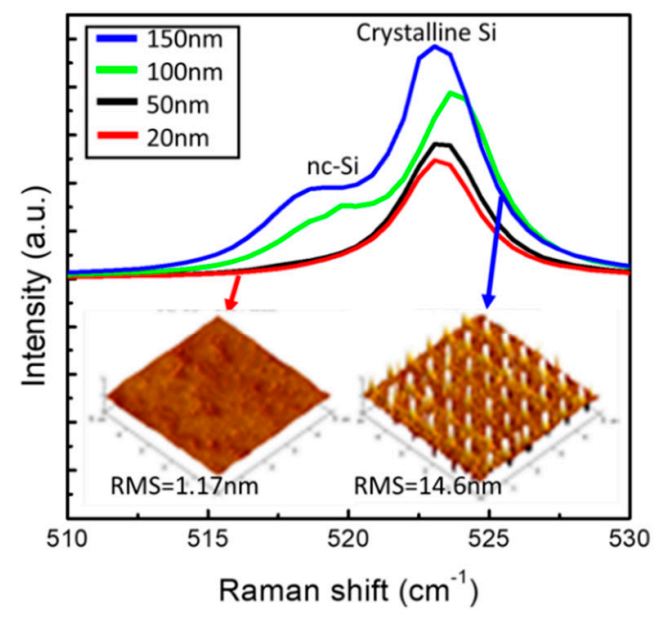

(f)

Figure 3. (a) SEM image of random grain growth poly-Si channel; (b) SEM image of a Si matrix using the LCG technique with the grain filter illustrated in the inset; (c) SEM image of a thin LCG Si film after CMP and SEECO etch (solution of $\mathrm{K}_{2} \mathrm{Cr}_{2} \mathrm{O}_{7}$ water mixed with HF) to reveal the grain boundaries; (d) SEM image of a single grain GAA Si NW FET intentionally located in a Si gain; (e) grain size distribution of a Si channel without the LCG technique and the inset is the image analysis conducted by the ImageJ software. (f) The Raman spectrums of the Si channel before and after the CMP thinning process and inset AFM images show the related surface morphology and the root mean square roughness. 


\subsection{Gate-All-Around Si Nanowire FET Fabrication}

Ultra-thin-body (UTB) silicon in an insulator (SOI) configuration has been widely studied and adopted in our previous works [26-29] for its outstanding electrostatics due to excellent suppression of the short channel effects (SCEs). However, it is still hard to thoroughly remove the interface traps and fixed oxide defects in back oxide which inevitably degrade the device performance [30,31]. For the recrystallized poly-Si channel, this issue becomes more serious, because the fast quench speed causes more interface defects between the poly-Si and buried oxide interface. Here, a configuration evolution from UTB to GAA was achieved by proposing a whole chemical process to suspend the single-grain Si NW and to eliminate the interface traps and fixed oxide defects.

By precisely controlling the soaking time of dilute HF that consumes sacrificial oxide and buried oxide, we can simply obtain a single-grain GAA Si NW FET. Figure 4a shows a tilted SEM image of a single-grain GAA Si NW FET with a multi-nanowire channel in order to increase the total driving current. The active region of the FET was intentionally designed and fabricated within a single Si grain. The number of nanowires depends on the grain size and the limitation of the lithographic process. For example, we may put an $8 \times 4$ static random-access memory (SRAM) mini-array within a grain with a size of $1.04 \times 0.83 \mu \mathrm{m}$ following the 7-nm-node IC design rule [32,33]. For the transistor beyond a 5-nm-node, we may put more FETs or even functional units within a Si grain.

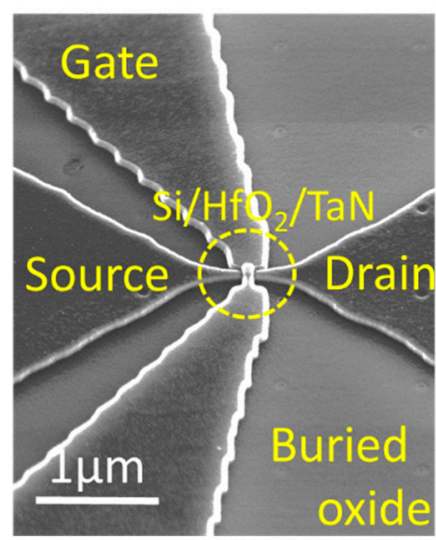

(a)

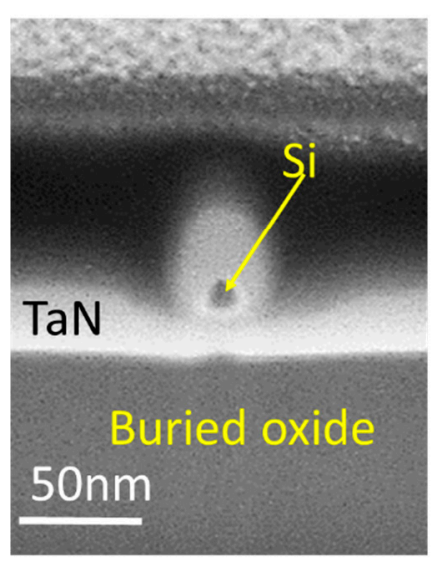

(d)

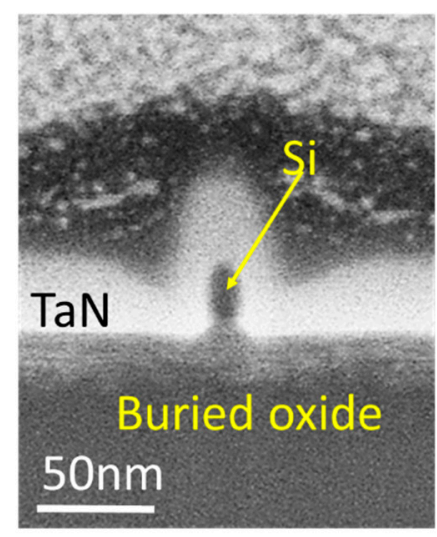

(b)

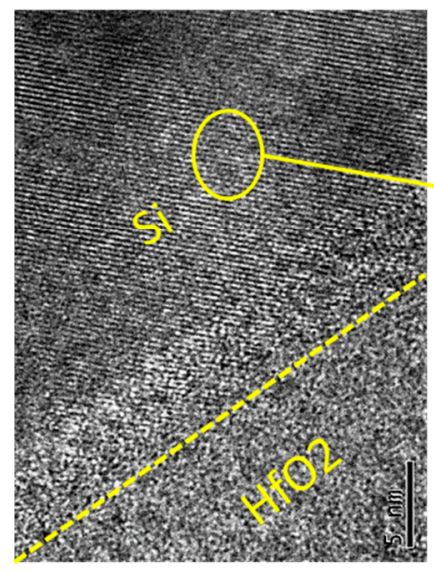

(e)

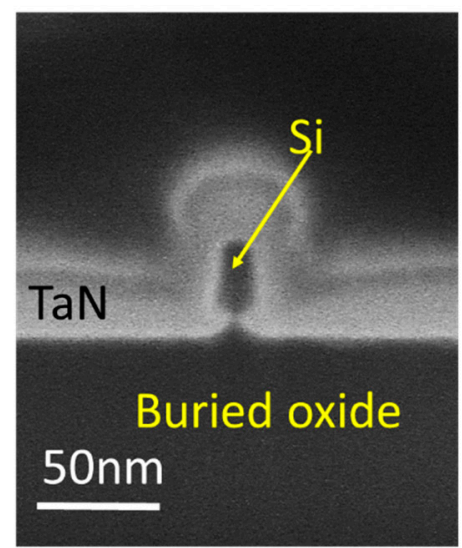

(c)

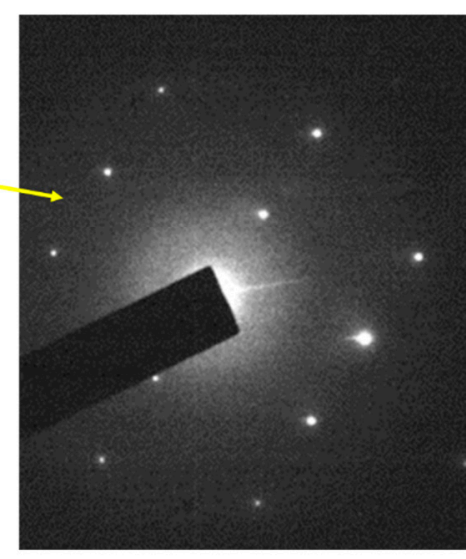

(f)

Figure 4. (a) A tilted SEM image of a multi-wire GAA Si NW FET. The configurations of (b) tri-gate, (c) $\Omega$-gate, and (d) GAA Si FET with different soaking times in the channel fabrication step. (e) A HRTEM image of single-grain $\mathrm{Si}$ and $\mathrm{HfO}_{2}$ dialectic layer. (f) A selected area diffraction pattern (SADP) from Si. 
A progressive evolution of device configuration fabricated by increasing HF soaking time is exhibited in Figure $4 \mathrm{~b}-\mathrm{d}$. An $\Omega$-gate Si FET was demonstrated while the buried oxide underneath was partially etched away, as shown in Figure 4c. The coverage of the HK/MG stack can be increased to enhance controllability by a longer soaking time to totally detach the buried oxide that touches the $\mathrm{Si}$ channel. The ultimate GAA Si NW FET with $W_{\text {fin }} / H_{\text {fin }} / L_{\text {gate }}=15 \mathrm{~nm} / 15 \mathrm{~nm} / 30 \mathrm{~nm}$ was achieved by 5 min soaking time as presented in Figure $4 \mathrm{~d}$. The high-resolution transmission electron microscopy (HRTEM) image in Figure 4e exhibits an ALD-deposited $\mathrm{HfO}_{2}$ and a single-grain Si lattice. The selected area diffraction pattern (SADP) for the laser-crystallized channel in Figure $4 \mathrm{f}$ reveals that the Si channel is highly recrystallized.

\subsection{Highly Activated Ultra-Shallow-Junction Formed by FIR-LA}

One of the bottle-necks in monolithic 3D GAA Si NW FET devices is to form highly activated (low sheet resistance $\mathrm{R}_{\mathrm{sh}}$ ) source/drain extensions with abrupt, ultra-shallow junctions (USJ) [34,35]. How to repair the amorphized surface bombarded by dopant species as well as to avoid severe dopant diffusion that causes the SCEs during the high-energy activation and heat dissipation through the ILD downward to the bottom metal interconnect and device/circuits become a key challenge.

The introduced FIR-LA is an intraband excitation process [36,37]. It can selectively transfer energies to the free carriers and repairs defects or activates the dopants without damaging the top HK/MG nano-structure of a gate-first device. The limited heating zone and short process duration result in fast heat dissipation and therefore keep the bottom metal interconnects and the device/circuit "cool".

A high-resolution transmission electron microscope (HRTEM) image of a Si after the dopant implantation process is shown in Figure 5a and clearly indicates that the surface is bombarded into disorder. The amorphous Si layer is about 15-nm-thin, appearing after the $\mathrm{P}^{31+}$ dopant implantation at a heavy dosage of $5 \times 10^{15} \mathrm{~cm}^{-2}$ and $5 \mathrm{KeV}$ ion energy. With an FIR-LA condition of $125 \mathrm{~W}$ laser power for $100 \mu \mathrm{s}$ at $350{ }^{\circ} \mathrm{C}$ annealing temperature, the amorphous layer shall be epitaxial regrown. Figure $5 \mathrm{~b}$ shows the dopant profiles with and without the FIR-LA using a secondary ion mass spectrometer (SIMS) and they are compared to conventional rapid thermal annealing (RTA). Apparently, a $37.6 \mathrm{~nm}$ diffusion depth at the level of $10^{18}$ dopant concentration was observed in conventional RTA at $800{ }^{\circ} \mathrm{C}$ for $10 \mathrm{~s}$ annealing time which increases the risk of current leakage between source and drain due to lateral diffusion in the nanodevices. In contrast, the FIR-LA contributes only a $7.3 \mathrm{~nm}$ diffusion depth which reflects the fact that a short-pulse and long-wavelength laser annealing effectively suppresses the SCEs. The series resistance in the inset table, which is another index of the annealing process and dominates the final performance of a scaling device, was further reduced from 213 to $93 \mathrm{Ohm} / \gamma$ by adopting the FIR-LA to replace conventional RTA. The nearly diffusionless dopant profiles and low resistivity represent the advantage of such a low-thermal budget FIR-LA in monolithic 3D-ICs.

\subsection{Hybrid Laser-Assisted Salicidation}

Nickel salicidation is a crucial process for advanced MOS transistors for its low Si consumption, low formation temperature, and low line width sheet resistance dependence as compared to cobalt or titanium silicide [18,19]. Previous studies proved that a rough $\mathrm{NiSi}$ film and $\mathrm{NiSi}_{2}$ facet (111) formed during conventional two-step RTA, which will lead to Fermi-level pining and a junction spiking leakage current $[38,39]$. Additionally, the second step of conventional two-step RTA usually reaches 400 to $600{ }^{\circ} \mathrm{C}$ to form a low-resistivity NiSi film, which is slightly higher than the BEOL CMOS process requirement that may cause a reliability issue for monolithic stacking $[9,10]$. Here, we introduce a hybrid laser-assisted salicidation process, that is first annealed at $250{ }^{\circ} \mathrm{C}$ for $30 \mathrm{~s}$ in an RTA system followed by second-step FIR-LA with $100 \mu$ s process time at room temperature $\left(\mathrm{T}_{\text {sub }}=25^{\circ} \mathrm{C}\right)$, to form a thin and uniform Si-rich $\mathrm{NiSi}_{x}$ film as well as to suppress the formation of $\mathrm{NiSi}_{2}$ facet (111).

The X-ray diffraction (XRD) patterns of nickel silicide formed by the hybrid laser-assisted salicidation process were displayed in Figure 6a. Five nickel monosilicide (NiSi) peaks, corresponding to the $(002)$,

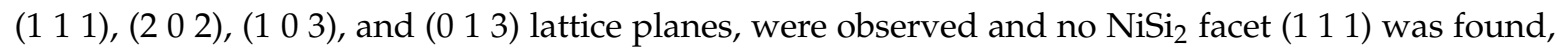


which promises a high-quality and low-resistivity NiSi film. The morphology of the NiSi film prepared by the hybrid laser-assisted salicidation process was analyzed by high-resolution transmission electron microscopy (HRTEM). The second-step FIR-LA melts the high-resistivity $\mathrm{Ni}_{2} \mathrm{Si}$ phase near the surface and the melt front diffuses downward to the $\mathrm{Si}$ and $\mathrm{Ni}_{2} \mathrm{Si}$ interface. The mixing of $\mathrm{Ni}_{2} \mathrm{Si}$ and $\mathrm{Si}$ occurs via liquid phase diffusion [38], which leads to a uniform NiSi film without heterogeneous aggregation and spike at the interface between the NiSi and the $\mathrm{Si}$, which is confirmed in Figure $6 \mathrm{~b}, \mathrm{c}$. The atomic percentage of $\mathrm{Si}$, identified by energy-dispersive $\mathrm{X}$-ray spectroscopy (EDS), slightly raises from 49.87 (point 1) to $54.74 \%$ (point 3), implying $\mathrm{Ni}_{2} \mathrm{Si}$ diffused from the surface to the bottom.

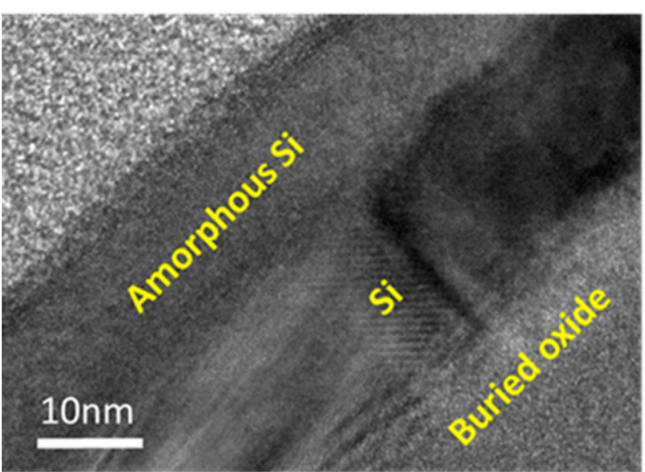

(a)

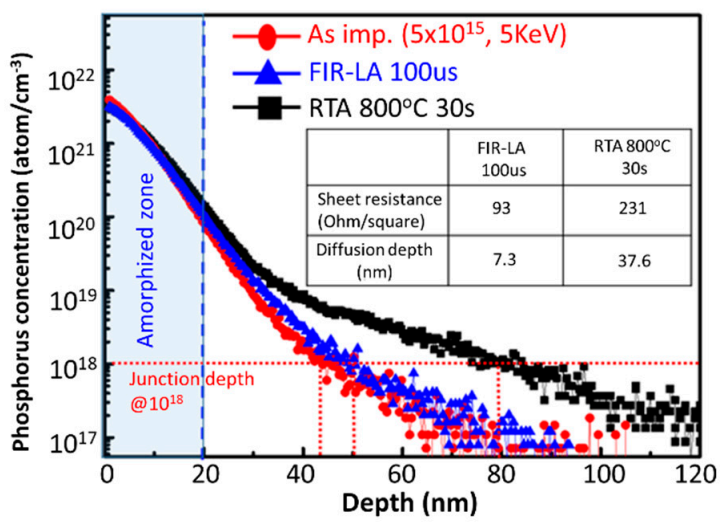

(b)

Figure 5. (a) A HRTEM image of Si after dopant implantation. (b) Dopant profiles characterized by a secondary ion mass spectrometer (SIMS). The inset table presents the sheet resistance differences between FIR-LA and RTA.

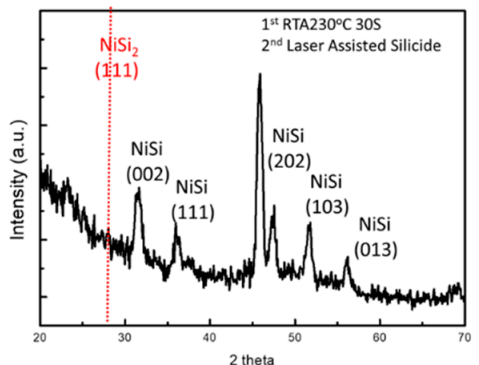

(a)

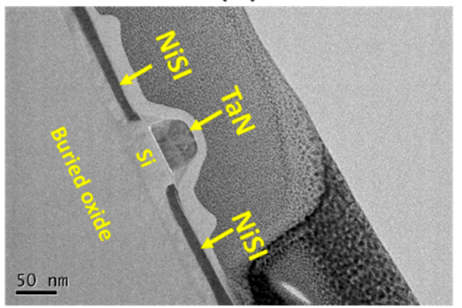

(b)

\begin{tabular}{|c|c|c|c|}
\hline Spectrum Label & Location 1 & Location 2 & Location3 \\
\hline $\mathrm{Si}$ & 49.87 & 54.08 & 54.74 \\
\hline $\mathrm{Ni}$ & 50.13 & 45.92 & 45.26 \\
\hline Total (atomic \%) & 100.00 & 100.00 & 100.00 \\
\hline
\end{tabular}

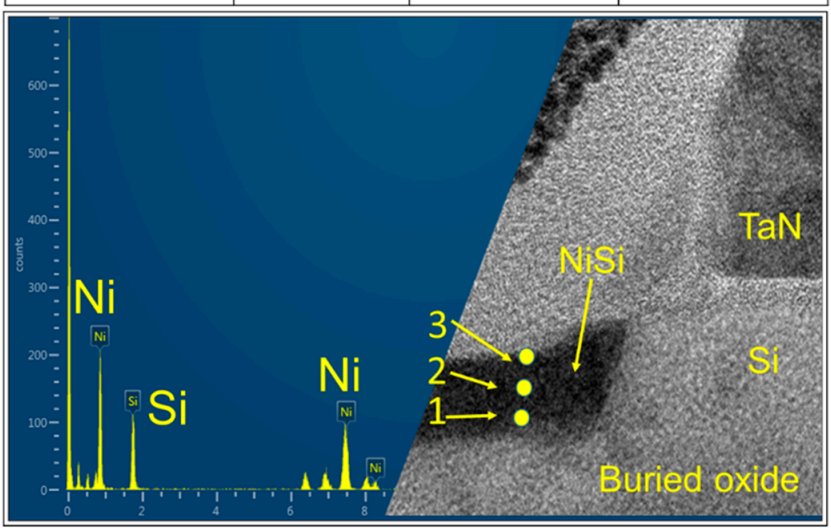

(c)

Figure 6. (a) The XRD patterns of the nickel silicide formed by the hybrid laser-assisted salicidation; (b) a TEM image of a Si FET with a flat and uniform NiSi film in the source and drain region; (c) energy- dispersive $\mathrm{X}$-ray spectroscopy (EDS) information of the NiSi film in the source and drain region at various depth.

\subsection{Device Uniformity Characterization and FIR-LA Validation for Monolithic Three Dimension Integrated} Circuits Application

Figure 7a provides the transfer characteristics at $\left|\mathrm{V}_{\mathrm{d}}\right|=1 \mathrm{~V}$ of the $\Omega$-gate Si FETs and the GAA Si NW FETs with $W_{\text {fin }} / \mathrm{H}_{\text {fin }} / \mathrm{L}_{\text {gate }}=15 \mathrm{~nm} / 15 \mathrm{~nm} / 30 \mathrm{~nm}$ simultaneously using location-controlled-grain technique. 
The $\Omega$-gate Si FETs exhibits subthreshold swing (S.S. 71 mV/dec.), driving current $291 \mu \mathrm{A} / \mu \mathrm{m}$ (n-type) and $274 \mu \mathrm{A} / \mu \mathrm{m}$ (p-type) at $\mathrm{V}_{\text {th }} \pm 0.8 \mathrm{~V}$. The single-grain GAA Si NW FETs have better gate controllability and therefor exhibits superior electrical properties with steeper subthreshold swing (S.S.) of about $65 \mathrm{mV} / \mathrm{dec}$, higher driving currents (Ion) of $327 \mu \mathrm{A} / \mu \mathrm{m}$ (n-type) and $297 \mu \mathrm{A} / \mu \mathrm{m}$ (p-type) at $\mathrm{V}_{\text {th }} \pm 0.8 \mathrm{~V}$, and higher on/off current ratio $\left(\mathrm{I}_{\mathrm{on}} / \mathrm{I}_{\mathrm{off}}\right)$ of $>10^{5}$. The off currents of the GAA Si NW FETs, extracted from the curves at $\mathrm{V}_{\mathrm{g}}=0 \mathrm{~V}$, reach about $1 \mathrm{nA} / \mu \mathrm{m}$ which was much lower than the $\Omega$-gate Si FETs.

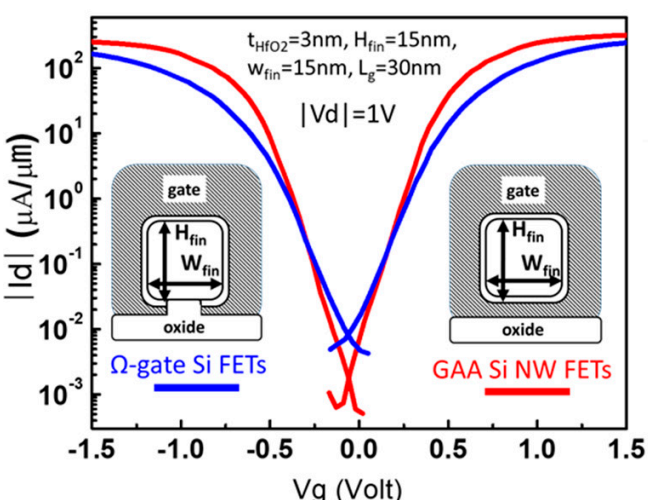

(a)

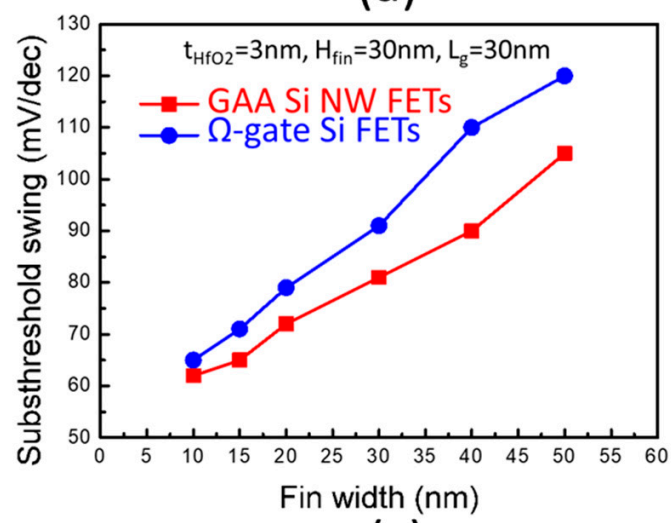

(c)

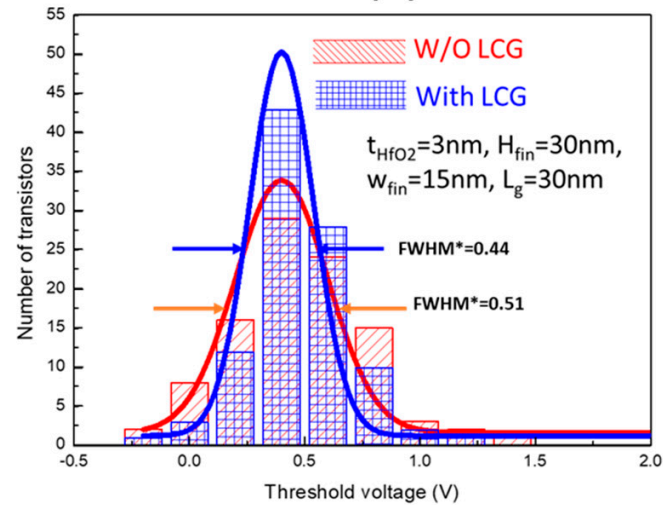

(e)

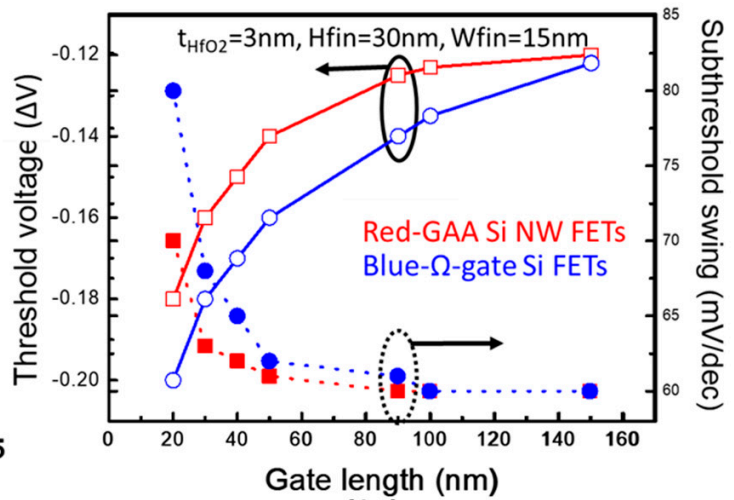

(b)

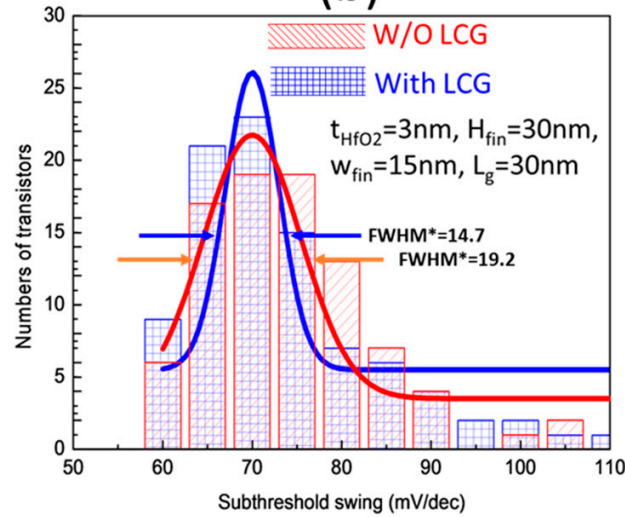

(d)

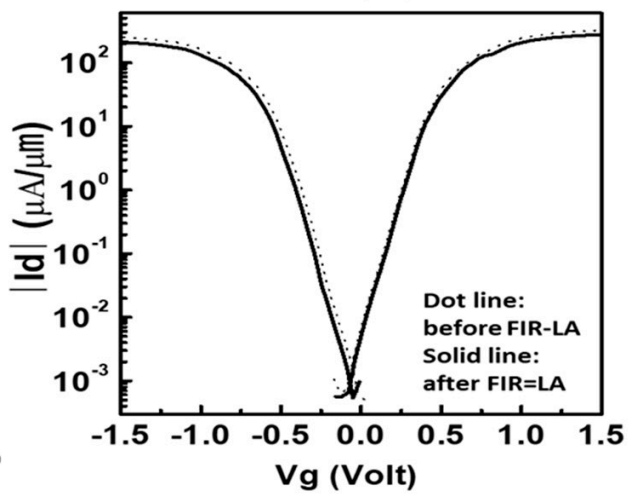

(f)

Figure 7. (a) A comparison of transfer characteristics between $\Omega$-gate Si FETs and GAA Si NW FETs; (b) the dependencies of threshold voltage and S.S. on the transistor gate length $\left(\mathrm{Lg}_{\mathrm{g}}\right)$; (c) the dependency of S.S. on the fin width $\left(\mathrm{W}_{\text {Fin }}\right)$; the distributions of (d) S.S. and (e) $\mathrm{V}_{\text {th }}$ of the GAA Si NW FETs with and without the LCG technique; (f) the transfer characteristics of a GAA Si NW FET before and after the FIR-LA. 
To extract the threshold voltage $\left(\mathrm{V}_{\mathrm{th}}\right)$ and S.S. dependencies of these two types of single-grain transistor on the gate length $\left(\mathrm{Lg}_{\mathrm{g}}\right)$, the device structures having a similar size were chosen for comparison. The data were collected and plotted in Figure $7 \mathrm{~b}$. According to the result, the $\mathrm{V}_{\text {th }}$ decrease while the $\mathrm{Lg}_{\mathrm{g}}$ becomes shorter. Further, the S.S. is found to be increased with the shrinkage in $\mathrm{Lg}$. It indicates that the SCEs dominate the output characteristics at sub- $100 \mathrm{~nm}$ of both transistors. The slower $\mathrm{V}_{\text {th }}$ roll-off and S.S. ramp up rate in the line graph of the GAA Si NW FET elucidate that the gate-all-around configuration has better ability in suppressing SCEs. This evidence can be found in Figure $7 \mathrm{c}$ as well. The S.S. rises from 65 to $121 \mathrm{mV} / \mathrm{dec}$. ( $\Delta$ S.S. $=56 \mathrm{mV} / \mathrm{dec}$.), while the $\mathrm{W}_{\text {Fin }}$ increases from 10 to $50 \mathrm{~nm}$ for the $\Omega$-gate Si FETs. Comparatively, the GAA Si NW FETs reveals a lower S.S. in the same $\mathrm{W}_{\mathrm{Fin}}$ and a smaller $\Delta$ S.S. ( $43 \mathrm{mV} / \mathrm{dec}$.).

By introducing the LCG technique for monolithic 3D-ICs, we can prevent the electrical property variation caused by the random grain size and the unpredictable boundaries. Figure $7 \mathrm{~d}$,e show the cumulative plots of S.S. and $V_{\text {th }}$ of the GAA Si NW FETs with and without the LCG technique within an 8-inch Si wafer. Both plots demonstrate the single-grain GAA Si NW FETs with the LCG technique have smaller S.S. (from 19.2 to $14.7 \mathrm{mV} / \mathrm{dec}$ at FWHM) and $\mathrm{V}_{\text {th }}$ deviations (from 0.51 to $0.44 \mathrm{~V}$ at FWHM).

To validate the feasibility of the monolithic 3D-IC sequential integration process, the transfer characteristics of a GAA Si NW FET with a two-layer metal interconnect (M1-M2) were prepared and tested before and after FIR-LA. Evidently, the lack of obvious changes in the $\mathrm{I}_{\mathrm{d}}-\mathrm{V}_{\mathrm{g}}$ behaviors before and after the FIR-LA in Figure $7 \mathrm{f}$ ensures the compatibility of the monolithic 3D-IC sequential integration process.

\section{Conclusions}

In summary, novel methodologies to fabricate GAA Si NW FETs with high performance and low power consumption were reported. By integrating the location-controlled-grain technique, laser crystallization, far-infrared laser dopant activation, and hybrid laser-assisted salicidation, sub-50 nm GAA Si NW FETs with lower sensitivity to Vth roll-off and subthreshold swing degradation as the gate length scaled down were presented. Furthermore, electrical validation results provide convincing evidence for the feasibility of monolithic three-dimensional integrated circuits. The advanced 3D architecture demonstrated in this article enables high-bandwidth sequentially stackable circuits to achieve superior performance and low power consumption for future mobile and neuromorphic applications.

Author Contributions: Conceptualization, T.-Y.H. and C.-C.Y.; methodology, T.-Y.H. and P.-Y.H.; software, P.-Y.H.; validation, T.-Y.H., T.-Y.H. and C.-C.Y.; resources, C.-H.S. and J.-M.S.; data curation, T.-Y.H. and C.-C.Y.; writingoriginal draft preparation, T.-Y.H.; writing—review and editing, T.-Y.H. and C.-C.Y.; supervision, W.-K.Y. and M.-C.W.; project administration, W.-K.Y. and M.-C.W.; funding acquisition, C.-C.Y. All authors have read and agreed to the published version of the manuscript.

Funding: The authors would like to thank the Ministry of Science and Technology (MOST 108-2221-E-492-010-) and National Applied Research Laboratories (NARLabs) of the Republic of China for financial support.

Conflicts of Interest: The authors declare no conflict of interest.

\section{References}

1. Schaller, R. Moore's law: Past, present and future. IEEE Spectr. 1997, 34, 52-59. [CrossRef]

2. Mack, C. Fifty years of Moore's law. IEEE Trans. Semicond. Manuf. 2011, 24, 202-207. [CrossRef]

3. Katti, G.; Stucchi, M.; De Meyer, K.; Dehaene, W. Electrical modeling and characterization of through silicon via for three-dimensional ICs. IEEE Trans. Electron Devices 2010, 57, 256-262. [CrossRef]

4. Lee, M.; Wu, S.; Yang, M.; Chen, K.; Luo, G.; Lee, L.; Kao, M. High-performance Poly-Si TFTs using ultrathin HfSiOx gate dielectric for monolithic three-dimensional integrated circuits and system on glass applications. IEEE Electron Device Lett. 2010, 31, 824-826. [CrossRef]

5. Chen, H.; Chang, C.; Lu, N.; Wu, J.; Han, M.; Cheng, Y.; Wu, Y. Characteristics of gate-all-around junctionless Poly-Si TFTs with an ultrathin channel. IEEE Electron Device Lett. 2013, 34, 897-899. [CrossRef] 
6. Shulaker, M.; Hills, G.; Park, R.; Roger, T.; Howe, K.; Saraswat, H.; Wong, S.M. Three-dimensional integration of nanotechnologies for computing and data storage on a single chip. Nature 2017, 547, 74-78. [CrossRef]

7. Levinson, J.; Shepherd, F.R.; Scanlon, P.J.; Rider, M. Conductivity behavior in polycrystalline semiconductor thin film transistors. J. Appl. Phys. 1982, 53, 1193. [CrossRef]

8. Chalmers, B. High-speed growth of sheet crystals. J. Cryst. Growth 1984, 70, 3-10. [CrossRef]

9. Sameshima, T. Laser crystallization for large-area electronics. Appl. Phys. A 2009, 96, 137-144. [CrossRef]

10. Chen, T.; Yeh, C.; Lou, J. Effects of grain boundaries on performance and hot-carrier reliability of excimer-laser annealed polycrystalline silicon thin film transistors. J. Appl. Phys. 2004, 95, 5788-5794. [CrossRef]

11. Vandooren, A.; Franco, J. 3-D sequential stacked planar devices featuring low-temperature replacement metal gate junctionless top devices with improved reliability. IEEE Trans. Electron Devices 2018, 65, 5165-5171. [CrossRef]

12. Schwarzenbach, W.; Maleville, C.; Daval, N. Advanced FD-SOI and beyond low temperature SmartCut ${ }^{\mathrm{TM}}$ enables high density 3-D SoC applications. IEEE J. Electron Devices Soc. 2019, 7, 863-868. [CrossRef]

13. Ishihara, R.; Danciu, D.; Tichelaar, F.; He, M.; Hiroshima, Y.; Inoue, S.; Shimoda, T.; Metselaar, J.W.; Beenakker, C.I.M. Microstructure characterization of location-controlled Si-islands crystallized by excimer laser in the $\mu$-Czochralski (grain filter) process. J. Cryst. Growth 2007, 299, 316-321. [CrossRef]

14. Ryoichi, I.; Artyom, B.; Paul, A. Location-control of large Si grains by dual-beam excimer-laser and thick oxide portion. Jpn. J. Appl. Phys. 2000, 39, 7.

15. Ryoichi, I.; Paul, C.; Barry, D.; Metselaar, C. Property of Single-Crystalline Si TFTs Fabricated with $\mu$-Czochralski (grain filter) Process. SPIE Proc. 2003, 5004, 10-19.

16. Rajendran, B.; Shenoy, R.; Witte, D.; Chokshi, N.; DeLeon, R.; Tompa, G.; Pease, R. Low thermal budget processing for sequential 3-D IC fabrication. IEEE Trans. Electron Devices 2007, 54, 707-714. [CrossRef]

17. Yang, C.; Huang, W.; Hsieh, T.; Wu, T.; Wang, H.; Shen, C.; Yeh, W.; Shiu, J.; Chen, Y.; Wu, M.; et al. High gamma value 3D-Stackable HK/MG-Stacked Tri-Gate Nanowire Poly-Si FETs with embedded source/drain and back gate using low thermal budget green nanosecond laser crystallization technology. IEEE Electron Device Lett. 2016, 37, 533-536. [CrossRef]

18. Deng, F.; Johnson, A.; Asbeck, P.; Lau, S. Salicidation process using NiSi and its device application. J. Appl. Phys. 1997, 81, 8047. [CrossRef]

19. Colgana, E.; Gambinoa, J.; Hongb, Q. Formation and stability of silicides on polycrystalline silicon. Mater. Sci. Eng. R Rep. 1996, 16, 43-96. [CrossRef]

20. Johnson, M.; Biegelsen, D.; Moyer, M. Deuterium passivation of grain-boundary dangling bonds in silicon thin films. Appl. Phys. Lett. 1982, 40, 882. [CrossRef]

21. Scheller, L.; Weizman, M.; Simon, P.; Fehr, M.; Nickela, N. Hydrogen passivation of polycrystalline silicon thin films. J. Appl. Phys. 2012, 112, 63711. [CrossRef]

22. Ahn, M.; Takuya Saraya, T.; Kobayashi, M.; Sawamoto, N.; Ogura, A.; Hiramoto, T. superior subthreshold characteristics of gate-all-around P-Type Junctionless Poly-Si nanowire transistor with ideal subthreshold slope. Jpn. J. Appl. Phys. 2020, 59, 7. [CrossRef]

23. Mohammad, M.; Kees, B. Stacking of single-grain thin-film transistors. Jpn. J. Appl. Phys. 2009, 48, $3 \mathrm{~S} 2$.

24. IahihR, R.; Matsumura, M. Ultra-large grain growth of Si films on glassy substrate. Electron Lett. 1995, 31, 22.

25. Aaron, M.; Voutsas, T.; Raj, S. A systematic study and optimization of parameters affecting grain size and surface roughness in excimer laser annealed polysilicon thin films. J. Appl. Phys. 1997, 82, 4302.

26. Wu, T.; Huang, W.; Yang, C.; Chen, H.; Hsieh, T.; Lin, W.; Kao, M.; Chen, C.; Yao, J.; Jian, Y.; et al. High performance and low power monolithic Three-Dimensional Sub-50 nm Poly Si Thin film transistor (TFTs) circuits. Sci. Rep. 2017, 7, 1368. [CrossRef]

27. Huang, W.; Shieh, J.; Kao, M.; Shen, C.; Huang, T.; Wang, H.; Yang, C.; Hsieh, T.; Hsieh, J.; Yu, P.; et al. Enabling N-Type Polycrystalline Ge Junctionless FinFET of Low thermal budget by in situ doping of channel and visible pulsed laser annealing. Appl. Phys. Express 2017, 10, 026502. [CrossRef]

28. Huang, W.; Shieh, J.; Pan, F.; Yang, C.; Shen, C.; Wang, H.; Hsieh, T.; Wu, S.; Wu, M. Charge-trap non-volatile memories fabricated by laser-enabled low-thermal budget processes. Appl. Phys. Lett. 2015, 107, 183506. [CrossRef]

29. Yang, C.; Hsieh, T.; Huang, W.; Shen, C.; Shieh, J.; Yeh, W.; Wu, M. Recent progress in low-temperature-process monolithic three dimension technology. Jpn. J. Appl. Phys. 2018, 57, 04FA06. [CrossRef] 
30. Ouisse, T.; Cristoloveanu, S.; Borel, G. Hot-carrier-induced degradation of the back interface in short-channel silicon-on-insulator MOSFETS. IEEE Electron Device Lett. 1991, 12, 290-292. [CrossRef]

31. Ouisse, T.; Cristoloveanu, S.; Borel, G. Electron trapping in irradiated SIMOX buried oxides. IEEE Electron Device Lett. 1991, 12, 312-314. [CrossRef]

32. Lawrence, T.; Vinay, V.; Greg, Y. ASAP7: A 7-nm FinFET predictive process design Kit. MicroElectron J. 2016, 53, 105-115.

33. Tapas, D.; Girish, P.; Yogesh, C. Performance Evaluation of 7-nm node negative capacitance FinFET-based SRAM. IEEE Electron Device Lett. 2017, 38, 1161-1164.

34. Singh, K.; Kumar, S.; Goel, E.; Singh, B.; Singh, P.; Baral, K.; Kumar, H.; Jit, S. Effects of source/drain elevation and side spacer dielectric on drivability performance of non-abrupt Ultra Shallow junction gate underlap GAA MOSFETs. Indian J. Phys. 2017, 92, 171-176. [CrossRef]

35. Yang, B.; Buddharaju, K.; Teo, S.; Singh, N.; Lo, G.; Kwong, D. Vertical silicon-nanowire formation and Gate-All-around MOSFET. IEEE Electron Device Lett. 2008, 29, 791-794. [CrossRef]

36. Langfeld, R.; Baumann, H.; Bethge, K.; Jex, H. CO2-Laser Induced annealing and diffusion in high energy As-ion-implanted silicon. Phys. Lett. A 1981, 82, 148-150. [CrossRef]

37. James, R. Pulsed CO2 laser annealing of silicon. Semicond. Semimet 1984, 23, 555-623.

38. Mia, S.; Jiaa, C.; Zhaob, Q.; Mantlb, S.; Urban, K. NiSi2/Si interface chemistry and epitaxial growth mode. Acta Mater. 2009, 57, 232-236. [CrossRef]

39. Chena, H.; Linb, C.; Huang, C.; Chien, C. The effect of pulsed laser annealing on the nickel silicide formation. Microelectron. Eng. 2010, 87, 2540-2543. [CrossRef]

(C) 2020 by the authors. Licensee MDPI, Basel, Switzerland. This article is an open access article distributed under the terms and conditions of the Creative Commons Attribution (CC BY) license (http://creativecommons.org/licenses/by/4.0/). 